\title{
(อ) OPEN ACCESS \\ International consensus conference on stool banking for faecal microbiota transplantation in clinical practice
}

\author{
Giovanni Cammarota (D) , ${ }^{1}$ Gianluca Ianiro, ${ }^{2}$ Colleen R Kelly, ${ }^{3}$ Benjamin H Mullish (D) , \\ Jessica R Allegretti, ${ }^{5}$ Zain Kassam, ${ }^{6,7}$ Lorenza Putignani, ${ }^{8}$ Monika Fischer, ${ }^{9}$ \\ Josbert J Keller, ${ }^{10,11}$ Samuel Paul Costello, ${ }^{12}$ Harry Sokol, ${ }^{13,14,15}$ Patrizia Kump, ${ }^{16}$ \\ Reetta Satokari, ${ }^{17}$ Stacy A Kahn, ${ }^{18}$ Dina Kao, ${ }^{19}$ Perttu Arkkila, ${ }^{20}$ Ed J Kuijper, ${ }^{21}$ \\ Maria J GT Vehreschild, ${ }^{22}$ Cristina Pintus, ${ }^{23}$ Loris Lopetuso, ${ }^{24}$ Luca Masucci, ${ }^{25}$ \\ Franco Scaldaferri, ${ }^{24}$ E M Terveer, ${ }^{11,21}$ Max Nieuwdorp, ${ }^{26}$ Antonio López-Sanromán, ${ }^{27}$ \\ Juozas Kupcinskas, ${ }^{28}$ Ailsa Hart, ${ }^{29}$ Herbert Tilg, $^{30}$ Antonio Gasbarrini ${ }^{31}$
}

For numbered affiliations see end of article.

\section{Correspondence to}

Professor Giovanni Cammarota, Internal Medicine and Gastroenterology, Fondazione Policlinico A Gemelli IRCCS, Roma 00168, Italy;

giovanni.cammarota@unicatt.it

$\mathrm{GC}$ and $\mathrm{Gl}$ are joint first authors.

Received 26 July 2019

Revised 10 September 2019

Accepted 22 September 2019

Published Online First

28 September 2019

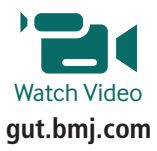

Check for updates

(C) Author(s) (or their employer(s)) 2019. Re-use permitted under CC BY-NC. No commercial re-use. See rights and permissions. Published by BMJ.

To cite: Cammarota $\mathrm{G}$ laniro G, Kelly $C R$, et al. Gut 2019:68:2111-2121.

\section{ABSTRACT}

Although faecal microbiota transplantation (FMT) has a well-established role in the treatment of recurrent Clostridioides difficile infection (CDI), its widespread dissemination is limited by several obstacles, including lack of dedicated centres, difficulties with donor recruitment and complexities related to regulation and safety monitoring. Given the considerable burden of CDI on global healthcare systems, FMT should be widely available to most centres.

Stool banks may guarantee reliable, timely and equitable access to FMT for patients and a traceable workflow that ensures safety and quality of procedures. In this consensus project, FMT experts from Europe, North America and Australia gathered and released statements on the following issues related to the stool banking: general principles, objectives and organisation of the stool bank; selection and screening of donors; collection, preparation and storage of faeces; services and clients; registries, monitoring of outcomes and ethical issues; and the evolving role of FMT in clinical practice,

Consensus on each statement was achieved through a Delphi process and then in a plenary face-to-face meeting. For each key issue, the best available evidence was assessed, with the aim of providing guidance for the development of stool banks in order to promote accessibility to FMT in clinical practice.

\section{INTRODUCTION}

Faecal microbiota transplantation (FMT) has a well-established role in the treatment of recurrent Clostridioides difficile infection (CDI). ${ }^{1-13}$ FMT working protocols have undergone considerable advancements in recent years, including the use of frozen faeces, ${ }^{2}$ capsules ${ }^{6}$ and the release of guidelines to provide methodological guidance. ${ }^{14-17}$

Despite these improvements, the dissemination of FMT has been limited owing to lack of dedicated centres, difficulties with donor recruitment, regulatory and safety concerns. Given the increasing burden of CDI, ${ }^{18} 19$ the provision of FMT should be widely and rapidly accessible. Moreover, as recently highlighted in a warning from the Food and Drug
Administration (FDA), FMT requires strict quality control to prevent harmful consequences. ${ }^{20}$

Stool banks can provide reliable, timely and equitable access to FMT for CDI, and also facilitate a standardised, cost-effective and traceable workflow that ensures safety and quality of procedures ${ }^{21}$ compared with single FMT centres. Stool banks are currently unevenly distributed and differ considerably in legislation, organisation and structure..$^{22-24}$

The aim of this consensus report is to provide guidance on the general organisation and the criteria required to establish a stool bank.

\section{METHODS}

\section{Consensus development process}

The consensus process was developed according to the following steps: selection of expert panel members, identification of key issues and related working group (WG), development of statements based on best available evidence, achievement of consensus through the Delphi technique and a faceto-face final meeting.

Twenty-nine consensus members, with documented expertise in the field of FMT and stool banking, took part in the expert panel. Based on personal expertise, each member was assigned to one of six WGs: general principles, objectives and organisation of the stool bank; selection and screening of donors; collection, preparation and storage of faeces; services and clients; registries, monitoring of outcomes, and ethical issues; and update on FMT in clinical practice. Each WG proposed a list of key issues and developed statements related to the assigned topic(s).

For each key issue, the best available evidence was obtained through a systematic review of the pertinent literature. If the related statements were suitable for Grading of Recommendations Assessment, Development and Evaluation (GRADE) assessment, based on PICO (population, intervention, comparator, outcome) questions, they have been graded accordingly. ${ }^{2526}$ Otherwise, statements were released only as expert opinions. 
The elaborated statements were uploaded to an online voting system (http://armstrong.wharton.upenn.edu/delphi2/) ${ }^{27}$ and disseminated to the panel. Experts' responses were collected, addressed and shared with the panel after each round. For each statement, experts were requested to rate their level of agreement: (1) agree strongly; (2) agree with reservation; (3) undecided; (4) disagree; (5) disagree strongly.

Consensus was achieved if at least $80 \%$ of respondents expressed a strong agreement or an agreement with reservation. Statements that did not pass this threshold were revised and rated again in further voting rounds, until consensus was reached.

Panel experts gathered in Rome on 22 June 2019 for refinement and final approval of the statements.

\section{RESULTS}

Three rounds of voting were necessary to reach consensus for all statements. Respectively, $73 \%$ of statements passed the $80 \%$ agreement threshold after the first round, 90\% after the second round, and 100\% after the third round. Overall, the expert panel released 40 statements. Of these, 17 were developed according to the GRADE approach, and 23 were based on expert opinion (EO). For statements developed through the GRADE approach, the certainty of evidence (CoE) and the strength of recommendation (SoR) were indicated.

\section{General principles, objectives and general organisation of the stool bank}

(1) Statement (EO): Stool banks aim to safely collect, store and distribute faeces from screened healthy donors that have been processed and stored, in order to treat CDI through FMT. Stool banks may also participate in research protocols under strict investigative conditions.

Comment: The principal aim of the stool bank is the provision of FMT to healthcare centres to treat recurrent or severe CDI in a timely, safe and traceable manner. Stool banks would relieve hospitals from the administrative burdens of individual FMT donor programmes and enhance the standardisation of FMT. ${ }^{28}$

(2) Statement (EO): Stool banks should guarantee high levels of security in the processing of patients' and donors' personal data, according to national or higher-level regulation.

Comment: Management of records related to all FMT steps should be regulated by local health organisations. This should ensure that the fundamental right to protection of personal data is fully guaranteed, following pertinent national and/or international legislation. ${ }^{29}$ All necessary measures should be taken to ensure that the data processed are kept confidential and secure and that donors and recipients are not identifiable.

(3) Statement (EO): The director of the stool bank should fulfil the following criteria: medical microbiologist or infectious disease specialist or gastroenterologist with scientific and clinical experience in the field of FMT.

The director should ensure (a) that the stool bank primarily functions for the provision of faecal suspensions for the treatment of CDI; (b) that the material used is consistently collected, controlled, processed, stored and distributed, pursuant to applicable rules and regulations; (c) donor-recipient traceback; (d) participation in research protocols under strict investigative conditions.

Comment: The panel suggests that the stool bank director should have had specialty training in gastroenterology, microbiology or infectious diseases (as CDI is usually managed within these specialties) and should have specific expertise in FMT. The director should guarantee that the bank provides faecal suspensions in clinical practice only to treat CDI, and that participation in research protocols is accepted only for rigorously designed and ethically approved studies. Moreover, he/she must ensure that the stool bank respects pertinent legislation and that traceability of bank processes is guaranteed.

(4) Statement (EO): The director of the stool bank should appoint the members of the scientific committee, who must have experience in the field of FMT, for clinical, regulatory and scientific advice. The scientific committee and the director should then appoint (a) a responsible physician, who coordinates individual requests for access to the bank from accredited healthcare facilities; (b) a physician(s), who evaluates, selects and recruits stool donors; (c) a microbiologist and/or pharmacist, who coordinates all the processes connected to the processing of faeces in the laboratory, up to storage; (d) a biobanking expert, who appropriately stores faeces under standardised conditions; (e) a director of quality management to ensure compliance with the various procedural steps.

Comment: Together, the director and the scientific committee should appoint appropriate professional figures to oversee all fundamental processes of the stool bank, including the recruitment of donors and the processing and storage of faeces according to existing regulatory requirements or certification systems.

(5) Statement (EO): In the absence of other appropriate guidance (eg, local directives), the scientific committee should establish protocols to direct the following duties: (a) evaluation and approval of requests from healthcare facilities; (b) regular review of the healthcare provider eligibility requirements; (c) regular analysis of stool bank activities; (d) analysis of reported adverse events (AEs).

Comment: FMT is not specifically regulated in all countries. ${ }^{21}$ Local FMT programmes ${ }^{30}$ remain relatively rare worldwide. In the absence of dedicated local directives or direction from regulatory authorities, the scientific committee should be in charge of specific tasks, described in the statement.

(6) Statement (EO): Stool banks should undergo appropriate approval from the relevant national regulatory authorities of each country.

Comment: Some countries/regions regulate FMT as a drug, some as a tissue, some via 'hybrid' regulation, and others do not provide specific regulation. Specifically, the USA UK, and France regulate FMT as a medicinal product. ${ }^{31}$ The FDA has demonstrated evolution of their regulatory position on FMT over time, and exercise a policy of enforcement discretion regarding investigational new drug requirements where FMT is used for CDI not responding to standard treatment. ${ }^{32}$ In 2014, the European Commission expressed an opinion that FMT is a 'combined product', given the presence of both human cells and non-human components (eg, microbial genes) within the material; however, given that the human cellular components of FMT are not generally believed to be the active components, they have decided that FMT falls outside of the European Tissues and Cells Directives, and have deferred regulation to different European countries for national/local regulation. ${ }^{33}$ The Therapeutic Goods Administration in Australia are currently formulating the regulatory framework for FMT in that country. ${ }^{34}$ The expert panel agreed that all stool banks should continue to operate under the designated regulatory authority for each country. 


\section{Selection and screening of donors}

(7) Statement (EO): Stool donation must be voluntary. Donors should be informed about the potential risks and benefits of donating and provide appropriate written informed consent.

Comment: Apart from the health screening, stool donors do not directly benefit from donating. Stool donation is significantly different from other live tissue or cell-based donations as it is not invasive. Personnel recruiting donors should explain in detail the screening process and the measures taken to ensure donor confidentiality. Potential donors should be made aware that they can withdraw from this process at any time. ${ }^{35-38}$

(8) Statement (EO): Stool donors may receive financial compensation for their time and travel expenses or a reimbursement that surpasses these expenses. Individual applicability of these concepts should follow local regulations.

Comment: There is longstanding debate about the provision of compensation for tissue or cell-based donors. ${ }^{39-41}$ In many regions (eg, Europe), direct compensation for tissue or cell-based donations is not permitted. In the USA, compensation for donation of some non-organ human material (eg, plasma or sperm) is permitted.

As compared with other types of donation, stool donation occurs much more frequently. Accordingly, there is a significant burden on stool donors, including logistical issues and restrictions on behaviour. Moreover, data from a large stool bank suggest high rates of donor drop out owing to major time commitments. ${ }^{42}$ The panel suggests that compensation and/or reimbursement may be provided if permitted by appropriate regulatory guidance.

(9) Statement: Recruitment of donors should be through a continuous, structured workflow to prevent shortages.

$\mathrm{CoE}$ : very low; SoR: weak

Comment: Among 330 physicians who did not offer FMT, $80 \%$ reported difficulties with the logistics of preparation/ delivery, and $45 \%$ cited complexity and cost of donor screening as being prohibitive. ${ }^{43}$ Stool banks have emerged to facilitate access to FMT material as compared with patient-directed donation, but donor recruitment can be challenging. Observational data from a large stool bank suggest a high-rate of drop out and health-related exclusion from initial contact to stool donor qualification. ${ }^{42}$ Others small academic stool banks from Australia, Canada, USA and Denmark have reported similar challenges in procuring healthy donors. ${ }^{28}{ }^{44-47}$ Accordingly, we recommend dedicated donor operational personnel who use a structured workflow to ensure access to material.

(10) Statement: Undirected donors are recommended for stool bank recruitment. Under exceptional circumstances, patient-selected donors may be used if both the patient and donor understand the risks, benefits and alternatives.

CoE: low; SoR: strong

Comment: Evidence suggests that there is no significant difference in CDI cure rates between patient-selected and undirected healthy donors. ${ }^{1-7} 48$ However, head-to-head randomised controlled trials (RCTs) comparing these two types of donors have not been conducted.

Patient-selected donors were more frequently used in early FMT studies. However, the use of healthy undirected donors is essential for a stool bank, and has significant advantages, including prevention of shortages, reduction of screening-related expenses, a higher traceability, and a reduced likelihood of confidentiality concerns. ${ }^{49}$

(11) Statement: The medical history of candidate donors has to be evaluated, at the beginning of the screening process, by a
Box 1 Key issues to select potential donors at the preliminary interview

Known history of, or risk factors for, infectious diseases

- History of HIV, hepatitis B or C viruses, syphilis, human Tlymphotropic virus I and II

- Current systemic infection

- Use of illegal drugs

- High-risk sexual behaviour

- Previous tissue/organ transplant

- Recent hospitalisation or discharge from long-term care facilities

- High-risk travel/engaged in medical tourism

- Recent ( $\leq 6$ months) needle stick accident

- Recent ( $\leq 6$ months) body tattoo, piercing, earring, acupuncture

- Recent ( $\leq 2$ months) enteric pathogen infection

- Recent ( $\leq 2$ months) acute gastroenteritis with or without confirmatory test

- History of receiving growth hormone, insulin from cows or clotting factor concentrates

- Recent ( $\leq 2$ months) history of vaccination with a live attenuated virus, if there is a possible risk of transmission

Disorders potentially associated with perturbation of the gut microbiota

- Personal history of chronic gastrointestinal disease, including functional gastrointestinal disorders; inflammatory bowel disease; coeliac disease; other chronic gastroenterological diseases

- Personal history of systemic autoimmune disorders

- Personal history of cancer, including gastrointestinal cancers or polyposis syndrome

- Recent abnormal gastrointestinal symptoms (eg, diarrhoea, haematochezia, etc)

- Personal history of neurological/neurodegenerative disorders

- Personal history of psychiatric/neurodevelopmental conditions

- Obesity (body mass index >30) and/or metabolic syndrome/ diabetes

- First-degree family history of premature colon cancer or firstdegree family history of early onset polyposis syndromes

Drugs that can alter gut microbiota

- Recent ( $\leq 3$ months) exposure to systemic antimicrobial drugs, immunosuppressant agents, chemotherapy

- Chronic treatment ( $\geq 3$ months) with daily use of proton pump inhibitors

clinical questionnaire examining the risk of infectious diseases, the history of disorders potentially associated with perturbation of the gut microbiota and the use of treatments that can affect the gut microbiota (box 1).

CoE: moderate; SoR: strong

Comment: The purpose of the questionnaire is to minimise the risk of transferring infections or adverse gut microbiota 'traits'. When these or similar exclusion criteria are used, there have been few reported AEs related to the infusion of donor faeces. ${ }^{1-11}$

Since increasing age has been associated with altered gut microbiota composition, young individuals (aged $<50$ years or $<60$ if they have completed appropriate bowel cancer screening) are preferred as potential donors. Importantly, although the panel 


\section{Box 2 Donor blood and stool testing}

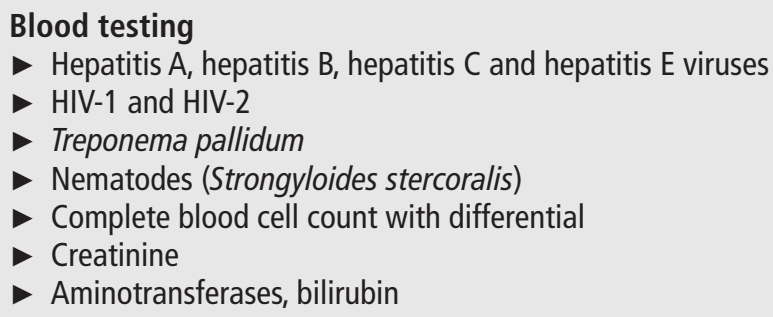

- Antibiotic-resistant bacteria (ARB), including vancomycinresistant Enterococci, meticillin-resistant Staphylococcus aureus), Gram-negative ARB including extended-spectrum $\beta$-lactamase-producing Enterobacteriaceae, and carbapenemresistant Enterobacteriaceae/carbapenemase-producing Enterobacteriaceae

- Norovirus, rotavirus, adenovirus

- Giardia lamblia, Cryptosporidium spp, Isospora and Microsporidia

- Protozoa and helminths/va and parasites (including Blastocystis hominis and Dientamoeba fragilis)

- Helicobacter pylori faecal antigen (for upper route of FMT delivery)

recommends exclusion of candidates with a personal history of systemic autoimmune diseases, the evidence is insufficient to exclude donors with atopy, asthma or food/seasonal allergies. Additionally, the panel recommends excluding candidates with a personal history of cancer but would consider donors with a history of non-malignant skin cancers (eg, basal cell carcinoma) following appropriate treatment. Although some stool banks exclude healthcare workers with exposure to patients, available data suggest a low prevalence of antibiotic-resistant bacteria colonisation in this population. ${ }^{5051}$

Overall, data from a large USA stool bank ${ }^{42}$ suggest that nearly $90 \%$ of candidates are excluded after medical evaluation. Similar data have been presented by an Australian stool bank, where approximately $50 \%$ of donors are excluded after completion of the clinical questionnaire. ${ }^{44}$

(12) Statement: All candidate donors who have passed the medical interview must undergo blood and stool testing to exclude potentially transmittable diseases. Recommended tests are summarised in box 2 .

CoE: moderate; SoR: strong

Comment: There is relevant heterogeneity of donor screening across FMT studies and screening tests were largely incomplete in $>50 \%$ of published studies. ${ }^{52}$ The panel believes that it is indispensable to include a screening panel that should constitute a reference standard operating procedure (SOP) of stool banks.

Here the testing recommendations from the previous consensus report were updated. ${ }^{15}$ The panel recommends the use of validated standard of care test methods (eg, culture, ELISA, PCR, serology) according to nationally and locally approved guidelines. Given the low percentage of qualified donors ${ }^{42}$ and the mental effect of non-meaninful incidental findings among healthy donors, we recommend a pragmatic approach to testing.
For blood testing, cytomegalovirus (CMV) and Epstein-Barr virus (EBV) should be considered. However, there is a high prevalence of prior exposure to both viruses in many healthy adult donors, and there are no reported cases of CMV- or EBVassociated disease attributable to faecal transplants performed in FMT centres, even among immunocompromised individuals. Accordingly, the panel recommends that immunocompromised recipients at risk for $\mathrm{CMV} / \mathrm{EBV}$-associated disease must be tested for these viruses before undergoing FMT and, if seronegative, appropriate conversation about risk, benefits and alternatives (including patient-selected donor use) should be considered. Importantly, we do not recommend exclusion of donors who are either CMV and/or EBV IgG positive. Consideration may be given to testing for active $\mathrm{CMV}(\mathrm{IgM})$ as an alternative approach.

The panel also recommends that Strongyloides stercoralis should always be tested for but that other nematodes (eg, Ascaris lumbricoides, Taenia solium and Taenia hominis) could be considered for testing, based on the physician's decision consistent with clinical, social and geographical features of the donors.

C-reactive protein may be considered in blood testing as it may be useful for identifying an underlying inflammatory state. However it is a non-specific inflammatory marker and its use in healthy, asymptomatic individuals is limited and potentially prone to incidental findings.

Consideration of stool testing for astrovirus and sapovirus may be considered, though there is a paucity of data on its clinical usefulness in healthy, asymptomatic donors. Additionally, faecal calprotectin may be considered: high calprotectin levels may be important if clinical symptoms are present, although its usefulness in healthy asymptomatic adults remains unknown. Finally, recommendations from the recent FDA alert,${ }^{20}$ about screening and testing of stool donors for multidrug-resistant organisms, have been incorporated in this statement (box 2).

(13) Statement: Donors should be given a questionnaire on the day of the donation to assess for any change in health that might have occurred since screening (box 3).

CoE: moderate; SoR: strong

Comment: To optimise safety and minimise risk, the panel recommends that donors complete a written questionnaire to assess for interval health change at each donation. This should include new symptoms, new sexual partners, high-risk travel and use of antibiotics or other drugs that might impair gut microbiota. This recommendation is supported by excellent safety data from several RCTs ${ }^{1-6}$ and meta-analyses. ${ }^{7-11}$

(14) Statement: Donors who repeatedly donate should undergo clinical assessment and a complete panel of laboratory testing every $8-12$ weeks.

CoE: very low; SoR: weak

Comment: As with other cell and tissue donation, it is important to ensure the continued safety of donated material even after the initial testing and approval of the donor. Faeces can theoretically be donated daily, and repeating a complete blood and stool screening at each donation would be unreasonable.

The panel proposed several measures to guarantee the safety of donor faeces. After initial testing, each donated faecal aliquot should be either (1) directly tested with a rapid molecular assay for stool pathogens or (2) remain in quarantine until that donor has passed a further donor screening at the end of a period of donation (even if the donor does not wish to provide faeces any more), and be available for administration to patients only after this further check.

(15) Statement (EO): Before starting the screening process, candidate donors should sign a written informed consent form including (a) acceptance of the screening process; (b) a 
Box 3 Issues to deal with on the same day of donation

to check for any recent-onset harmful events

- New Gl signs and/or symptoms (eg, diarrhoea, nausea, vomiting, abdominal pain, jaundice)

- New infection signs and/or symptoms (eg, fever or swollen lymph nodes)

- Use of antimicrobial agents or other drugs that might impair gut microbiota since the last screening

- Unprotected sexual encounters with new partners since the last screening

- High-risk or tropical travel or direct contact with human blood since the last screening
Box 4 General steps to be followed for the preparation

of frozen faecal suspensions

- A minimum quantity of $25 \mathrm{~g}$ of faeces for lower gastrointestinal delivery and $12.5 \mathrm{~g}$ of faeces for upper gastrointestinal delivery should be used for each sample.

- Before freezing, a cryoprotectant (eg, glycerol) should preferably be added up to a final concentration of $10 \%$.

- The final product must be put in a special sterile container, labelled, registered and stored at $-80^{\circ} \mathrm{C}$.

- On the day of faecal infusion, the faecal suspension should be thawed in a warm $\left(37^{\circ} \mathrm{C}\right.$ ) water bath or at room temperature, and infused within 6 hours from thawing.

of stool; ongoing certified laboratory testing; determination of quality control tests; and standards for release of the final product.

(19) Statement: General steps for the preparation and storage of frozen faecal suspensions must be followed (box 4). The formulation of faecal suspensions should be set up for specific delivery routes, based on local expertise, clinician and patient preference.

CoE: moderate; SoR: strong

Comment: The manipulation of donor faeces into frozen suspensions is an essential step in the workflow of the stool bank. A head-to-head RCT has shown similar efficacy of frozen and fresh FMT for the treatment of CDI. ${ }^{2}$

The suggested steps were slightly amended from our previous report. ${ }^{15}$ As FMT can be administered through different routes and each centre may have different expertise and confidence with them, the stool bank should be able to provide faecal suspensions in adequate formulations for each route requested by the recipient centres. The minimum quantity of donor faeces for lower gastrointestinal delivery has been decreased from $30 \mathrm{~g}$ to $25 \mathrm{~g}$, and $12.5 \mathrm{~g}$ for upper gastrointestinal delivery, as these quantities have documented success in large stool banks. ${ }^{57}$ Where upper gastrointestinal administration is considered most appropriate, both low-volume $(30 \mathrm{~mL})^{57}$ and high-volume $(500 \mathrm{~mL})^{58}$ preparations have been used, but there is still uncertainly about the ideal dose; caution is recommended owing to the reported risk of regurgitation and aspiration pneumonia with higher volumes. ${ }^{58}$ Capsule preparations should follow standard protocol. ${ }^{6}$ Further evidence on optimal dosing and formulation of capsules is required.

Finally, The $-80^{\circ} \mathrm{C}$ freezing temperature of the suspension and the use of glycerol as microbial cryopreservant have been used with success ${ }^{59} 60$ and were confirmed from our previous consensus. ${ }^{15}$

(20) Statement (EO): Containers of faecal suspensions should have a legible and indelible label that bears the unique code of the donor and the date of collection and processing. The code should be recorded in the recipient record/procedure report.

Comment: Faecal suspensions, in combination with a small portion of the original faeces or a small $(\sim 2 \mathrm{~mL})$ portion of the faecal suspension, must be stored under a unique donor code with a successive suffix number for donation time and date for retrospective quality assessment. Information on faecal suspension labels must include donor code, suspension number, production and expiration date, volume and storage temperature instructions.

storage of faeces must adhere to basic principles for safe preparation of human material, including strict working protocols to secure materials; maintenance of SOPs for the processing 
(21) Statement (EO): Stool banks should guarantee that all storage processes are carried out under controlled conditions and according to local, regional and national regulations.

Comment: Storage of faecal suspensions should be accommodated within the certified centralised biobanking facility in a specific $-80^{\circ} \mathrm{C}$ freezer with connected alarm notification to ensure storage of the material at appropriate temperature. The temperature range of the stored material and the duration of any out-of-range periods must be recorded and controlled. Products that are exposed to temperature excursions, which might affect the quality of the final product, must be discarded according to local, national and international procedures for the management of medical waste.

(22) Statement: The faecal suspension can be stored or up to 2 years. After this deadline, unused stored material must must be discarded according to local, national and international procedures for the management of medical waste.

CoE: very low ; SoR: weak

Comment: Reports from a large stool bank show that the use of faecal suspensions stored for up to 2 years does not undermine the clinical success of FMT for the treatment of CDI if samples are stored at $-80^{\circ} \mathrm{C}$. $^{426162}$ However, as a preclinical study reports a decline in microbial viability in frozen faeces after 9 months, ${ }^{63}$ the panel suggests it is preferable to use frozen samples within 1 year from donation.

\section{Services and clients}

(23) Statement (EO): Access to stool bank should be provided to physicians caring for patients with CDI. It cannot be provided directly to patients.

Comment: FMT has well-described potential drawbacks and recognised unknowns about its use, including potential risks related to invasive administration, a theoretical possibility for transfer of infection and limited long-term safety data. Thus, the expert panel considered that every FMT delivery requires administration, documentation and appropriate follow-up that can only safely occur under the direction of a physician. Consequently, the expert panel concluded that it was inappropriate for faecal suspensions to be provided directly to patients.

(24) Statement (EO): Recipient centres should satisfy a set of criteria to access stool bank services, including (a) public or private hospitals or nursing homes that have identified a qualified physician with experience in the field of FMT; (b) documentation of treatments carried out, including procedure reports, patient data, preparation, timing and administration of the material coming from the stool bank, and safety and efficacy results; (c) the team of centre must have undergone training in FMT. Moreover, recipient centres should be instructed on recommended FMT best practices, including thawing of faecal suspensions, delivery modalities, informed consent and follow-up.

Comment: The panel agreed that FMT should be performed under the supervision of an appropriately trained physician and suggested that a physician with prior experience within specialties including, but not limited to, gastroenterology or infectious disease/medical microbiology might be suitable for overseeing the care of patients receiving FMT. However, endoscopydelivered FMT should be performed only by trained endoscopists to reduce the risk of complications.

Although the use of FMT has become increasingly well established for the treatment of CDI within many teaching hospitals and academic centres, the panel noted that many physicians working outside these centres (including in primary care) have more limited knowledge of this technique. Thus, the panel concluded that there was a clear role for stool banks to educate healthcare professionals about FMT.

No data on the learning curve in FMT are available, but the panel's opinion was that physicians should have been trained by performing at least 10 FMT procedures before being considered to be trained.

(25) Statement (EO): Faecal samples provided by the stool bank must be used in the recipient centre according to the same working rules followed by the stool bank itself, and cannot be transported, sold or given to other centres or individuals.

Comment: FMT is not regulated in all countries and there might not be regulatory body to certify the banking facility. Nevertheless, the panel agreed on the importance of the use of a standardised protocol for the final aspects of preparation and administration of faecal suspensions for all recipient centres served by the same stool bank.

(26) Statement (EO): Costs of FMT should be based on a business case and budget impact analysis and subsequently, reimbursed by health insurance, which will vary from country to country.

Comment: In the context of CDI, FMT has consistently been shown to be cost-effective compared with other potential therapeutic options, even when evaluated in a variety of global healthcare settings. ${ }^{64}$ Nevertheless, in most cases funding of FMT services has been historically provided ad hoc by teaching hospitals/academic centres, and FMT services sometimes run with a financial deficit. ${ }^{65} 66$ For sustainability of stool banks, the panel agreed that a robust business case and budget impact analysis is required.

(27) Statement (EO): Stool banks should document the release of each aliquot for transport and its destination. Faecal suspensions should remain frozen during transport, typically by the use of dry ice. On arrival, the recipient centre should confirm and document the identity of each faecal sample and that it has remained frozen.

Comment: The material should remain frozen in transit, to minimise any potential perturbation of microbiota composition and/or functionality. A growing body of evidence demonstrates that length and temperature of storage of faecal suspensions and/or stool itself could influence the culturable microbiota and profiles of bacterially derived metabolites that have been associated with the efficacy of FMT, including short-chain fatty acids. 546768

(28) Statement: Unless the frozen faecal suspension is to be used imminently by the recipient centre, it should be transferred immediately to a freezer and kept for no more than 2 months at $-20^{\circ} \mathrm{C}$ or for 2 years at $-80^{\circ} \mathrm{C}$ or colder.

CoE: very low; SoR: weak

Comment: There are few data demonstrating that the storage temperature of faeces influences the composition and potential functionality of the microbiota. ${ }^{546768}$ The expert panel noted that many recipient centres may not have routine access to $-80^{\circ} \mathrm{C}$ freezers, and that a pragmatic, simple storage protocol for recipient centres is likely to increase accessibility and takeup. Storage of faecal suspensions at $-20^{\circ} \mathrm{C}$ for up to 30 days appeared not to reduce efficacy of FMT in the treatment of CDI compared with fresh material ${ }^{2}$; conversely, the Firmicutes/ Bacteroidetes ratio was significantly higher in faecal samples that had been frozen for more than 50 days compared with identical fresh samples. ${ }^{69}$ Collectively, based on expert opinion, faecal suspensions could safely be kept at $-20^{\circ} \mathrm{C}$ (or colder) for up to 2 months in freezers at recipient centres. ${ }^{14}$

(29) Statement (EO): Clear traceability should be present at all steps of the FMT supply chain (eg, by batch number labelling) 
from the take-up of donated faecal samples through transport and delivery to sites and patient administration.

Comment: Recipient centres should retain thorough records of all steps of the supply chain, from the stool bank through to the point of patient administration, on the grounds of robust clinical governance. In the case of infectious AEs, this would facilitate a 'look-back' exercise from samples/records at the stool bank to identify if the donor was the source of the infection. ${ }^{61}$

\section{Registries, monitoring of outcomes and ethical issues}

(30) Statement (EO): An aliquot of each stool donation should be kept frozen for future testing in the case of AEs, in keeping with regulatory guidance. If for any reason a stool bank ceases its activity, stored samples and related data will be transferred to another stool bank or bio-bank having the same rules and the donor will be notified of the change in placement. In the absence of this possibility, the sample will be discarded following rules governing disposal of biological waste.

Comment: Stool banks should store an aliquot of the donor faeces, or samples of all delivered faecal suspensions, to guarantee traceability in case of AEs.

(31) Statement (EO): Donor records, including results of the screening process and identities of donors, should be stored in an official registry according to local regulatory guidance.

Comment: Management of the records related to the FMT procedure should be regulated by local health authorities. FMT procedure and donors' and recipients' records should be stored for at least 10 years. This may differ according to local and national requirements, and longer storage time may be necessary. The records of the stool bank will provide access to the long-term safety data.

(32) Statement: Practitioners of FMT should monitor patients for severe AEs that may be attributable to the procedure, such as hospitalisation, death or transmission of infections. These should be reported at the institutional level and to applicable regulatory agencies.

CoE: low; SoR: strong

Comment: The safety of FMT is one of the greatest concerns related to its use. ${ }^{70}{ }^{71}$ Short-term AEs relate primarily to the method of delivery or to acute infections contracted from the donor. Severe AEs include the following: death; life-threatening event; hospitalisation; disability or permanent impairment; other important event, including serious infection transmission (eg, HIV or viral hepatitis). All AEs potentially related to FMT, excluding extremely minor ones (eg, transient constipation or bloating), should be registered.

(33) Statement (EO): Recipient registries may be useful to monitor FMT recipients for AEs.

Comment: The potential for long-term consequences of FMT is almost unknown. A priori knowledge is not available about the impact of transferring complex microbial communities. Animal studies have indicated that the composition of the gut microbiota can affect host susceptibility to diseases, ${ }^{72}$ and human studies have consistently shown that FMT durably alters the microbiome of the recipient. ${ }^{73} 74$ FMT is unlike other treatments in that it was rapidly adopted for widespread clinical use, bypassing the drug-development process, which typically collects prospective efficacy and safety data on large numbers of patients before making a treatment available. ${ }^{64} 75$ Therefore, it is important to collect real-world evidence for the short- and long-term safety of the procedure. In the USA and Europe, FMT recipient registries have been launched to track efficacy and safety outcomes in adult and paediatric patients after FMT. ${ }^{17} 7677$ In addition to infections, the possibility that gut microbiota associated with a disease phenotype (eg, obesity) will be transplanted and the risk for these chronic disorders in recipients must be assessed. Prospective registries enrolling large cohorts of recipients with long-term follow-up are the only practical method to achieve this aim. ${ }^{78}$

\section{Evolving role of FMT in clinical practice}

(34) Statement: FMT is highly recommended as a treatment option for multiply recurrent CDI

CoE: high; SoR: strong

Comment: FMT has a well-established role in the treatment of multiply recurrent CDI. This is typically defined as three or more confirmed episodes or two episodes that required hospitalisation. It has been found to be better than the standard of care antibiotics and placebo in several RCTs. ${ }^{1-6}$ In addition, several meta-analyses suggest that FMT can achieve nearly 90\% clinical cure rate for the treatment of recurrent CDI. ${ }^{7-11}$

(35) Statement: FMT is recommended as an option for severe CDI not responding to standard treatment.

CoE: moderate; SoR: strong

Comment: FMT has been shown to be an effective treatment for patients with severe CDI not responding to standard of care antibiotics (according to pertinent guidelines), regardless of the number of previous episodes. In several randomised and nonrandomised studies, sequential faecal infusion protocols were highly successful in patients with severe CDI. ${ }^{79-83}$

(36) Statement: FMT may be considered as a treatment option for fulminant CDI (defined as severe CDI plus hypotension or shock, ileus or toxic megacolon) in patients unfit for surgery.

CoE: very low; SoR: weak

Comment: Surgery is still considered the best option for patients with fulminant CDI not responding to maximum medical treatment. However, a subset of patients will be deemed unfit for surgery and in these cases, FMT can be considered as rescue therapy. ${ }^{79} 80$

(37) Statement: FMT could induce remission in mild-tomoderate ulcerative colitis (UC). Other potential therapeutic targets for FMT beyond CDI include metabolic syndrome, irritable bowel syndrome, hepatic encephalopathy, gut decolonisation of multidrug-resistant organisms, primary sclerosing cholangitis, autism and various roles in cancer. However, further studies are required before FMT can be recommended as a treatment option for any of these indications in clinical practice. To date, its use should be limited to the research setting or compassionate use, in the absence of alternative therapeutic options.

CoE: moderate; SoR: weak

Comment: Increasing evidence suggests that FMT could be a treatment option in other disorders beyond CDI. The most robust data come from studies in UC, as RCTs and meta-analyses show that FMT can induce clinical remission in nearly $30 \%$ of patients with mild-to-moderate UC, ${ }^{84-91}$ although data about maintenance of remission are still limited. ${ }^{92}$

The effect of FMT on irritable bowel syndrome has been investigated in several RCTs and in a meta-analysis, but the results are heterogeneous, and FMT cannot be recommended for this indication. ${ }^{93-96}$ Early evidence suggests that FMT might be a promising approach in hepatic encephalopathy, ${ }^{97} 98$ metabolic syndrome, ${ }^{99} 100$ decolonisation of multidrug-resistant bacteria, ${ }^{101-103}$ primary sclerosing cholangitis, ${ }^{104}$ autism, ${ }^{105}$ and in the treatment of cancer. ${ }^{106}{ }^{107}$ Nevertheless, many problems about the use of FMT in these non-CDI indications remain unresolved, including the identification, for each indication, 
of successful donor profiles, adequate working protocols and correct integration of FMT within the therapeutic management pathway of the disease.

Owing to the increasing media attention around microbiome research, many patients are seeking FMT for a wide variety of non-CDI indications, though there is limited evidence for efficacy in these. Physicians may feel pressured by these patients and unsure how to act, though the decision to recommend FMT should follow the same logic as the recommendation of any other medical treatment under development. In this situation, clinical trials offer the safest and most ethical treatment option. ${ }^{108} 109$ Related ethical issues should be discussed with patients. Those who do not have access to clinical trial participation for a condition in which there is some likelihood that FMT may provide benefit, and in the absence of alternative therapeutic options or where all available standard treatments have failed, may be offered FMT as compassionate use after appropriate informed consent and approval from a local ethics committee.

(38) Statement: FMT is a safe and effective treatment for CDI in children.

CoE: low; SoR: strong

Comment: There is sufficient evidence to support the safety and efficacy of FMT in children with CDI. ${ }^{110} 111$ CDI presents a growing health concern in young patients who are also more likely to have community-acquired CDI. Children who do not respond to standard antibiotic treatment, or who have recurrent or severe CDI, may be candidates for, and benefit from, FMT.

(39) Statement: The safety of FMT during pregnancy is unknown, therefore it should be avoided unless strictly needed.

CoE: very low; SoR: weak

Comment: To date there is insufficient evidence to evaluate clearly the safety of FMT during pregnancy. ${ }^{112}$ However, FMT could be used as a rescue treatment in pregnant women with overwhelming CDI if standard medical treatments are ineffective.

(40) Statement: FMT is generally safe in the short term ( $<1$ year), but plentiful data on long-term safety are still lacking. This fact should be discussed with patients and family and documented in the informed consent document.

CoE: low; SoR: weak

Comment: Some study including a small cohort of patients shows that FMT is safe at a 4 -year follow-up, ${ }^{74}$ but plentiful follow-up FMT trial data are still lacking. Trials are underway to collect data over a 10 -year follow-up period after FMT. ${ }^{113}$

\section{CONCLUSIONS}

This consensus report provides guidance on the general organisation and the criteria required to develop a stool bank. Several statements were presented as expert opinions and not following a GRADE approach owing to their intrinsically organisational or technical nature.

The expert panel identified roles and positions necessary to start stool banking activities, considered the issues of donation and donor recruitment/screening, and provided guidance on the preparation and storage of faeces. Guidelines on how to release faecal suspensions to centres and deal with safety and ethical concerns were established. Finally, in comparison with the previously published consensus conference, ${ }^{15}$ insufficient scientific evidence exists to support the use of FMT in clinical conditions other than the CDI, nor are data on long-term safety yet available. Despite the poor quality of evidence, a strong strength of recommendation is recognised in considering the option of FMT for treatment of children with CDI.
In conclusion, FMT should be accessible to all patients with CDI based on medical need with no interference from other factors, including site of hospitalisation, type of hospital or ability to pay. Given that the provision of FMT services is at present unequally distributed throughout certain countries, ${ }^{65} 66$ a stool bank would guarantee the dissemination of FMT worldwide and the accessibility of this potentially lifesaving procedure in an equitable fashion.

\section{Author affiliations}

${ }^{1}$ Internal Medicine and Gastroenterology, Day Hospital of Gastroenterology and Intestinal Microbiota Transplantation, Fondazione Policlinico A Gemelli IRCCS, Catholic University of Medicine, Roma, Italy

${ }^{2}$ Internal Medicine and Gastroenterology, Day Hospital of Gastroenterology and Intestinal Microbiota Transplantation, Fondazione Policlinico A Gemelli IRCCS, Roma, Italy

${ }^{3}$ Division of Gastroenterology, Alpert Medical School of Brown University, Providence, Rhode Island, United States of America

${ }^{4}$ Department of Metabolism, Digestion and Reproduction, Faculty of Medicine, Imperial College London, London, United Kingdom

${ }^{5}$ Division of Gastroenterology, Brigham and Women's Hospital, Harvard Medical

School, Boston, Massachusetts, United States of America

${ }^{6}$ Microbiome Informatics, Massachusetts Institute of Technology, Cambridge,

Massachusetts, United States of America

${ }^{7}$ OpenBiome, Somerville, Massachusetts, United States of America

${ }^{8}$ Parasitology Unit and Human Microbiome Unit, Bambino Gesù Pediatric Hospital,

Roma, Italy

${ }^{9}$ Department of Medicine, Indiana University, Indianapolis, Indiana, United States of America

${ }^{10}$ Department of Gastroenterologyand Hepatology, Haaglanden Medical Center,

2597 AX, The Hague, Netherlands

${ }^{11}$ National Donor Feces Bank, LUMC, Leiden, the Netherlands

${ }^{12}$ Department of Gastroenterology, The Queen Elizabeth Hospital, University of Adelaide, Woodville, South Australia, Australia

${ }^{13}$ Service de Gastroenterologie, Hôpital Saint Antoine, Sorbonne Université, Inserm,

Centre de Recherche Saint-Antoine, Paris, France

${ }^{14}$ French Group of Fecal Microbiota Transplantation, Paris, France

${ }^{15}$ INRA, UMR1319 Micalis, AgroParisTech, Jouy-en-Josas, France

${ }^{16}$ Division of Gastroenterology and Hepatology, Department of Internal Medicine

Medical University of Graz, Graz, Austria

${ }^{17}$ Human Microbiome Research Program, Faculty of Medicine, University of Helsinki,

Helsinki, Finland

${ }^{18}$ Division of Gastroenterology, Hepatology and Nutrition, Boston Children's Hospital,

Boston, Massachusetts, Uunited States of America

${ }^{19}$ Division of Gastroenterology, Department of Medicine, University of Alberta,

Edmonton, Alberta, Canada

${ }^{20}$ Department of Clinic of Gastroenterology, University of Helsinki and Helsinki

University Hospital, Helsinki, Finland

${ }^{21}$ Department of Medical Microbiology, Leiden University Medical Centre, Leiden, the Netherlands

${ }^{22}$ Department I of Internal Medicine; German Centre for Infection Research, Partner site Bonn-Cologne, University Hospital of Cologne, Cologne, Germany

${ }^{23}$ Tissues and Cells Area, Italian National Transplant Center, Rome, Italy

${ }^{24}$ Internal Medicine and Gastroenterology, Fondazione Policlinico A Gemelli IRCCS,

Roma, Italy

${ }^{25}$ Microbiology, Fondazione Policlinico Universitario Agostino Gemelli IRCCS, Catholic University of Medicine, Roma, Italy

${ }^{26}$ Department of Internal Medicine, Amsterdam University Medical Centers, location

AMC and VuMC, Amsterdam, Netherlands

${ }^{27}$ Gastroenterology and Hepatology Department, Hospital Universitario Ramon y

Cajal, Madrid, Spain

${ }^{28}$ Department of Gastroenterology, Institute for Digestive Research, Medical

Academy, Lithuanian University of Health Sciences, Kaunas, Lithuania

${ }^{29}$ Department of Gastroenterology, St Mark's Hospital, London, United Kingdom

${ }^{30}$ Department of Internal Medicine I, Gastroenterology, Endocrinology \& Metabolism, Innsbruck Medical University, Innsbruck, Austria

${ }^{31}$ Internal Medicine and Gastroenterology, Fondazione Policlinico A Gemelli IRCCS, Catholic University of Medicine, Roma, Italy

Twitter Giovanni Cammarota @GiovanniCammar9 and Gianluca laniro @ gianluca1aniro

Contributors GC conceived the idea and programme for the project. GC, and Gl organised and developed the project. GC, GI and CRK selected the expert panel and established the main topics. All panel members were involved in developing the statements with supporting evidence and drafted the text of discussion relevant 
to their statements. GC, GI, CRK, BHM, JRA, ZK and LP wrote the initial draft of the manuscript. All panel members read and revised the manuscript for important intellectual content and approved the final manuscript.

Funding The project was in part funded by the Catholic University of Rome, Line D-1 research funding.

Competing interests JRA received grants from, and consulted for, Finch Therapeutics and Merck and Co. GC, Gl, FS and LM received grants in the field of faecal microbiota transplantation (FMT) from the Italian Ministry of Health. SPC received fees from Shire, Ferring, Microbiotica, Pfizer and Janssen. MF is consultant to Finch Therapeutics Group and DSMB member for Rebiotix. AH has served as consultant, advisory board member or speaker for AbbVie, Atlantic, Bristol-Myers Squibb, Celltrion, Falk, Ferring, Janssen, MSD, Napp Pharmaceuticals, Pfizer, Pharmacosmos, Shire and Takeda; she also serves on the Global Steering Committee for Genentech. ZK is an employee/shareholder at Finch Therapeutics and advisor/ consultant at OpenBiome. JK and EJK received research grants from Vedanta Bioscences, Boston, USA. CPK is a clinical site for the PRISM FMT trial conducted by Finch Therapeutics. HS received unrestricted study grants: Danone, Biocodex, Enterome; board membership, consultancy or lecture fees: Carenity, Abbvie, Astellas, Danone, Ferring, Mayoly Spindler, MSD, Novartis, Roche, Tillots, Enterome, Maat, BiomX, Biose, Novartis, Takeda; co-funder of Exeliom Biosciences. All the remaining authors have nothing to declare.

Patient consent for publication Not required.

Provenance and peer review Not commissioned; externally peer reviewed.

Open access This is an open access article distributed in accordance with the Creative Commons Attribution Non Commercial (CC BY-NC 4.0) license, which permits others to distribute, remix, adapt, build upon this work non-commercially, and license their derivative works on different terms, provided the original work is properly cited, appropriate credit is given, any changes made indicated, and the use is non-commercial. See: http://creativecommons.org/licenses/by-nc/4.0/.

\section{ORCID iDs}

Giovanni Cammarota http://orcid.org/0000-0002-3626-6148

Benjamin H Mullish http://orcid.org/0000-0001-6300-3100

\section{REFERENCES}

1 van Nood E, Vrieze A, Nieuwdorp M, et al. Duodenal infusion of donor feces for recurrent Clostridium difficile. N Engl J Med 2013;368:407-15.

2 Lee $\mathrm{CH}$, Steiner T, Petrof EO, et al. Frozen vs fresh fecal microbiota transplantation and clinical resolution of diarrhea in patients with recurrent Clostridium difficile infection. JAMA 2016;315:142-9.

3 Kelly CR, Khoruts A, Staley C, et al. Effect of fecal microbiota transplantation on recurrence in multiply recurrent Clostridium difficile infection. Ann Intern Med 2016;165:609-16.

4 Cammarota G, Masucci L, laniro G, et al. Randomised clinical trial: faecal microbiota transplantation by colonoscopy vs. vancomycin for the treatment of recurrent Clostridium difficile infection. Aliment Pharmacol Ther 2015;41:835-43.

5 Hvas CL, Dahl Jørgensen SM, Jørgensen SP, et al. Fecal microbiota transplantation is superior to fidaxomicin for treatment of recurrent Clostridium difficile infection. Gastroenterology 2019;156:1324-32.

6 Kao D, Roach B, Silva M, et al. Effect of oral capsule- vs colonoscopy-delivered fecal microbiota transplantation on recurrent Clostridium difficile infection. JAMA 2017:318:1985-93

7 Kassam Z, Lee CH, Yuan Y, et al. Fecal microbiota transplantation for Clostridium difficile infection: systematic review and meta-analysis. AmJ Gastroenterol 2013;108:500-8.

8 Cammarota G, laniro G, Gasbarrini A. Fecal microbiota transplantation for the treatment of Clostridium difficile infection. J Clin Gastroenterol 2014;48:693-702.

9 Drekonja D, Reich J, Gezahegn S, et al. Fecal microbiota transplantation for Clostridium difficile infection: a systematic review. Ann Intern Med 2015;162:630-8.

10 Quraishi MN, Widlak M, Bhala N, et al. Systematic review with meta-analysis: the efficacy of faecal microbiota transplantation for the treatment of recurrent and refractory Clostridium difficile infection. Aliment Pharmacol Ther 2017;46:479-93.

11 laniro G, Maida M, Burisch J, et al. Efficacy of different faecal microbiota transplantation protocols for Clostridium difficile infection: a systematic review and meta-analysis. United European Gastroenterology Journal 2018;6:1232-44.

12 McDonald LC, Gerding DN, Johnson S, et al. Clinical practice guidelines for Clostridium difficile infection in adults and children: 2017 update by the infectious diseases Society of America (IDSA) and Society for Healthcare Epidemiology of America (SheA). Clin Infect Dis 2018;66:987-94.

13 Debast SB, Bauer MP, Kuijper EJ. European Society of clinical microbiology and infectious diseases. European Society of clinical microbiology and infectious diseases: update of the treatment guidance document for Clostridium difficile infection. Clin Microbiol Infect 2014;20(Suppl 2):1-26.

14 Mullish BH, Quraishi MN, Segal JP, et al. The use of faecal microbiota transplant as treatment for recurrent or refractory Clostridium difficile infection and other potential indications: joint British Society of Gastroenterology (BSG) and Healthcare Infection Society (HIS) guidelines. Gut 2018;67:1920-41.

15 Cammarota G, laniro G, Tilg H, et al. European consensus conference on faecal microbiota transplantation in clinical practice. Gut 2017:66:569-80.

16 Bakken JS, Borody T, Brandt LJ, et al. Treating Clostridium difficile infection with fecal microbiota transplantation. fecal microbiota transplantation workgroup. Clin Gastroenterol Hepatol 2011;9:1044-9.

17 Nicholson MR, Mitchell PD, Alexander E, et al. Efficacy of fecal microbiota transplantation for Clostridium difficile infection in children. Clin Gastroenterol Hepatol 2019;pii: S1542-3565:30427-6.

18 McGlone SM, Bailey RR, Zimmer SM, et al. The economic burden of Clostridium difficile. Clin Microbiol Infect 2012;18:282-9.

$19 \mathrm{Ma}$ GK, Brensinger CM, Wu Q, et al. Increasing incidence of multiply recurrent Clostridium difficile infection in the United States: a cohort study. Ann Intern Med 2017;167:152-8

20 Food and Drug Administration. Information pertaining to additional safety protections regarding use of fecal microbiota for transplantation - screening and testing of stool donors for multi-drug resistant organisms. Available: https://www. fda.gov/vaccines-blood-biologics/safety-availability-biologics/information-pertainingadditional-safety-protections-regarding-use-fecal-microbiota-transplantation [Accessed 1 Jul 2019].

21 Jørgensen SMD, Hvas CL, Dahlerup JF, et al. Banking feces: a new frontier for public blood banks? Transfusion 2019;59:2776-82.

22 Openbiome. Available: https://www.openbiome.org/ [Accessed 1 Jul 2019].

23 EnteroBiotix. Available: https://enterobiotix.com/ [Accessed 1 Jul 2019].

24 Terveer EM, van Beurden $\mathrm{YH}$, Goorhuis A, et al. How to: establish and run a stool bank. Clin Microbiol Infect 2017;23:924-30.

25 Guyatt GH, Oxman AD, Vist GE, et al. Grade: an emerging consensus on rating quality of evidence and strength of recommendations. BMJ 2008;336:924-6.

26 Robinson KA, Saldanha IJ, Mckoy NA. Frameworks for determining research gaps during systematic reviews. Agency for Healthcare Research and Quality 2011.

27 Hsu CC, Sandford BA. The Delphi technique: making sense of consensus. Pract Assess Res Eval 2007:12:1-6.

28 Costello SP, Tucker EC, La Brooy J, et al. Establishing a fecal microbiota transplant service for the treatment of Clostridium difficile infection. Clin Infect Dis 2016;62:908-14.

29 Directive 95/46/EC of the European Parliament and of the Council of 24 October 1995 on the protection of individuals with regard to the processing of personal data and on the free movement of such data. Official Journal $L ; 281: 0031-50$.

30 AGA center for gut microbiome research \& education. Available: http://www.gastro. org/about/initiatives/aga-center-for-gut-microbiome-research-education [Accessed 1 Jul 2019].

31 Cammarota G, Gallo A, laniro G, et al. Emerging drugs for the treatment of Clostridium difficile. Expert Opin Emerg Drugs 2019;24:17-28.

32 Enforcement policy regarding investigational new drug requirements for use of fecal microbiota for transplantation to treat Clostridium difficile infection not responsive to standard therapies; draft guidance for industry. Available: https:// www.federalregister.gov/documents/2016/03/01/2016-04372/enforcement-policyregarding-investigational-new-drug-requirements-for-use-of-fecal-microbiota-for [Accessed 1 Jul 2019].

33 Competent authorities on substances of human origin expert group (CASoHO E01718). Available: https://ec.europa.eu/health/sites/health/files/blood_tissues_ organs/docs/ev_20141203_sr_en.pdf [Accessed 1 Jul 2019].

34 Costello SP, Bryant RV. Faecal microbiota transplantation in Australia: bogged down in regulatory uncertainty. Intern Med J 2019;49:148-51.

35 WMA statement on organ and tissue donation. Available: https://www.wma.net/ policies-post/wma-statement-on-organ-and-tissue-donation/ [Accessed 1 Jul 2019].

36 Ethics Committee of the Transplantation Society. The consensus statement of the Amsterdam forum on the care of the live kidney donor. Transplantation 2004;78:491-2.

37 Gordon EJ. Informed consent for living donation: a review of key empirical studies, ethical challenges and future research. Am J Transplant 2012;12:2273-80.

38 Hays RE, LaPointe Rudow D, Dew MA, et al. The independent living donor advocate: a guidance document from the American Society of Transplantation's living donor community of practice (AST LDCOP). Am J Transplant 2015:15:518-25.

39 Friedman AL. Payment for living organ donation should be legalised. BMJ 2006:333:746-8

40 Payment for living organ donation should be legalised. Available: https://www.bmj. com/rapid-response/2011/10/31/payment-living-organ-donation-should-not-belegalised [Accessed 1 Jul 2019].

41 Adair A, Wigmore SJ. Paid organ donation: the case against. Annals 2011;93:191-2.

42 Kassam Z, Dubois NE, Ling K, et al. 512 - donor health screening for fecal microbiota transplantation: prospective evaluation of 15,317 candidate donors. Gastroenterology 2019;156:S-100-S-101.

43 Bakken JS, Polgreen PM, Beekmann SE, et al. Treatment approaches including fecal microbiota transplantation for recurrent Clostridium difficile infection (RCDI) among infectious disease physicians. Anaerobe 2013;24:20-4. 
44 Paramsothy S, Borody TJ, Lin E, et al. Donor recruitment for fecal microbiota transplantation. Inflamm Bowel Dis 2015;21:1600-6.

45 Craven LJ, Nair Parvathy S, Tat-Ko J, et al. Extended screening costs associated with selecting donors for fecal microbiota transplantation for treatment of metabolic syndrome-associated diseases. Open Forum Infect Dis 2017;4:ofx243.

46 Tariq R, Weatherly RM, Kammer PP, et al. Experience and outcomes at a specialized Clostridium difficile clinical practice. Mayo Clin Proc Innov Qual Outcomes 2017:1:49-56

47 Jørgensen SMD, Hansen MM, Erikstrup C, et al. Faecal microbiota transplantation: establishment of a clinical application framework. Eur J Gastroenterol Hepatol 2017:29:e36-45

48 Kim KO, Schwartz MA, Lin OST, et al. Reducing cost and complexity of fecal microbiota transplantation using universal donors for recurrent Clostridium difficile infection. Adv Ther 2019;36:2052-61.

49 Edelstein C, Daw JR, Kassam Z. Seeking safe stool: Canada needs a universal donor model. Can Med Assoc J 2016;188:E431-E432.

50 Decker BK, Lau AF, Dekker JP, et al. Healthcare personnel intestinal colonization with multidrug-resistant organisms. Clin Microbiol Infect 2018;24:82.e1-82.e4.

51 Carmeli Y, Venkataraman L, DeGirolami PC, et al. Stool colonization of healthcare workers with selected resistant bacteria. Infect Control Hosp Epidemiol 1998;19:38-40.

52 Lai CY, Sung J, Cheng F, et al. Systematic review with meta-analysis: review of donor features, procedures and outcomes in 168 clinical studies of faecal microbiota transplantation. Aliment Pharmacol Ther 2019:49:354-63.

53 Chu ND, Smith MB, Perrotta AR, et al. Profiling living bacteria informs preparation of fecal microbiota transplantations. PLoS One 2017;12:e0170922.

54 Miller JM, Astles R, Baszler T, et al. Biosafety blue ribbon panel; centers for disease control and prevention (CDC). guidelines for safe work practices in human and animal medical diagnostic laboratories. recommendations of a CDC-convened, biosafety blue ribbon panel. MMWR Supp/ 2012;61:1-102.

55 Good clinical laboratory practice (GCLP). World Health Organization on behalf of the special programme for research and training in tropical diseases, 2009. Available: http://www.who.int/tdr/publications/documents/gclp-web.pdf [Accessed 1 Jul 2019].

56 DAIDS guidelines for good clinical laboratory practice standards. 09 July, 2013. Available: http://www.niaid.nih.gov/LabsAndResources/resources/DAIDSClinRsrch/ Documents/gclp.pdf [Accessed 1 Jul 2019].

57 Osman M, O'Brien K, Stoltzner Z, et al. Safety and efficacy of fecal microbiota transplantation for recurrent Clostridium difficile infection from an international public stool bank: results from a 2050-patient multicenter cohort. Open Forum Infect Dis 2016;3(suppl_1).

58 Link A, Lachmund T, Schulz C, et al. Endoscopic peroral jejunal fecal microbiota transplantation. Dig Liver Dis 2016;48:1336-9.

59 Costello SP, Conlon MA, Vuaran MS, et al. Faecal microbiota transplant for recurrent Clostridium difficile infection using long-term frozen stool is effective: clinical efficacy and bacterial viability data. Aliment Pharmacol Ther 2015;42:1011-8.

60 Satokari R, Mattila E, Kainulainen V, et al. Simple faecal preparation and efficacy of frozen inoculum in faecal microbiota transplantation for recurrent Clostridium difficile infection - an observational cohort study. Aliment Pharmacol Ther 2015;41:46-53.

61 Elliott RJ, Nienga M, Ladha A, et al. Stool processing speed and storage duration do not impact clinical effectiveness of fecal microbiota transplantation across 1,924 Clostridium difficile infection patients. Am J Gastroenterol 2016;111.

62 Budree S, Elliott RJ, Rao S, et al. Donor stool processing time: the effect on the intestinal microbiome and clinical outcomes of fecal microbiota transplantation in Clostridium difficile infection. Gastroenterology 2017;152:S1006.

63 Jiang Z-D, Alexander A, Ke S, et al. Stability and efficacy of frozen and lyophilized fecal microbiota transplant (FMT) product in a mouse model of Clostridium difficile infection (CDI). Anaerobe 2017;48:110-4.

64 Le P, Nghiem VT, Mullen PD, et al. Cost-Effectiveness of competing treatment strategies for Clostridium difficile infection: a systematic review. Infect Control Hosp Epidemiol 2018:39:412-24.

65 Quraishi MN, Segal J, Mullish B, et al. National survey of practice of faecal microbiota transplantation for Clostridium difficile infection in the UK. J Hosp Infect 2017:95:444-5.

66 Mullish BH, Quraishi MN, Segal JP, et al. Introduction to the joint British Society of Gastroenterology (Bsg) and Healthcare Infection Society (His) faecal microbiota transplant guidelines. J Hosp Infect 2018;100:130-2.

67 Papanicolas LE, Choo JM, Wang Y, et al. Bacterial viability in faecal transplants: which bacteria survive? EBio Medicine 2019;41:509-16.

68 Gratton J, Phetcharaburanin J, Mullish BH, et al. Optimized sample handling strategy for metabolic profiling of human feces. Anal Chem 2016;88:4661-8.

69 Bahl MI, Bergström A, Licht TR. Freezing fecal samples prior to DNA extraction affects the Firmicutes to Bacteroidetes ratio determined by downstream quantitative PCR analysis. FEMS Microbiol Lett 2012;329:193-7.

70 Wang S, Xu M, Wang W, et al. Systematic review: adverse events of fecal microbiota transplantation. PLoS One 2016;11:e0161174.

71 Baxter M, Colville A. Adverse events in faecal microbiota transplant: a review of the literature. J Hosp Infect 2016;92:117-27.
72 Ridaura VK, Faith JJ, Rey FE, et al. Gut microbiota from twins discordant for obesity modulate metabolism in mice. Science 2013;341:1241214.

73 Li SS, Zhu A, Benes V, et al. Durable coexistence of donor and recipient strains after fecal microbiota transplantation. Science 2016;352:586-9.

74 Jalanka J, Mattila $\mathrm{E}$, Jouhten $\mathrm{H}$, et al. Long-term effects on luminal and mucosal microbiota and commonly acquired taxa in faecal microbiota transplantation for recurrent Clostridium difficile infection. BMC Med 2016;14:155.

75 Hecht GA, Blaser MJ, Gordon J, et al. What is the value of a food and drug administration investigational new drug application for fecal microbiota transplantation to treat Clostridium difficile infection? Clin Gastroenterol Hepatol 2014;12:289-91.

76 Kelly CR, Kim AM, Laine L, et al. The AGA's Fecal Microbiota Transplantation Nationa Registry: an important step toward understanding risks and benefits of microbiota therapeutics. Gastroenterology 2017;152:681-4.

77 Peri R, Aguilar RC, Tüffers K, et al. The impact of technical and clinical factors on fecal microbiota transfer outcomes for the treatment of recurrent Clostridioides difficile infections in Germany. United European Gastroenterol J 2019;7:716-22.

78 Gliklich RE DN. Registries for evaluating patient outcomes: a user's guide. Rockville, MD: AHRQ, 2010

79 Cammarota G, laniro G, Magalini S, et al. Decrease in surgery for Clostridium difficile infection after starting a program to transplant fecal microbiota. Ann Intern Med 2015:163:487-8.

80 Fischer M, Sipe B, Cheng Y-W, et al. Fecal microbiota transplant in severe and severecomplicated Clostridium difficile : a promising treatment approach. Gut Microbes 2017:8:289-302.

81 Fischer M, Sipe BW, Rogers NA, et al. Faecal microbiota transplantation plus selected use of vancomycin for severe-complicated Clostridium difficile infection: description of a protocol with high success rate. Aliment Pharmacol Ther 2015:42:470-6

82 Weingarden AR, Hamilton MJ, Sadowsky MJ, et al. Resolution of severe Clostridium difficile infection following sequential fecal microbiota transplantation. J Clin Gastroenterol 2013:47:735-7.

83 Ianiro G, Masucci L, Quaranta G, et al. Randomised clinical trial: faecal microbiota transplantation by colonoscopy plus vancomycin for the treatment of severe refractory Clostridium difficile infection-single versus multiple infusions. Aliment Pharmacol Ther 2018;48:152-9.

84 Moayyedi P, Surette MG, Kim PT, et al. Fecal microbiota transplantation induces remission in patients with active ulcerative colitis in a randomized controlled trial. Gastroenterology 2015;149:102-9.

85 Costello SP, Hughes PA, Waters 0 , et al. Effect of fecal microbiota transplantation on 8-week remission in patients with ulcerative colitis. JAMA 2019:321:156-64.

86 Paramsothy S, Kamm MA, Kaakoush NO, et al. Multidonor intensive faecal microbiota transplantation for active ulcerative colitis: a randomised placebocontrolled trial. The Lancet 2017·389:1218-28.

87 Rossen NG, Fuentes S, van der Spek MJ, et al. Findings from a randomized controlled trial of fecal transplantation for patients with ulcerative colitis. Gastroenterology 2015;149:110-8

88 Costello SP, Soo W, Bryant RV, et al. Systematic review with meta-analysis: faecal microbiota transplantation for the induction of remission for active ulcerative colitis. Aliment Pharmacol Ther 2017:46:213-24.

89 Narula N, Kassam Z, Yuan Y, et al. Systematic review and meta-analysis: fecal microbiota transplantation for treatment of active ulcerative colitis. Inflamm Bowel Dis 2017:23:1702-9.

90 Scaldaferri F, Pecere S, Petito V, et al. Efficacy and mechanisms of action of fecal microbiota transplantation in ulcerative colitis: pitfalls and promises from a first meta-analysis. Transplant Proc 2016;48:402-7.

91 Ianiro G, Bibbò G, Scaldaferri F, et al. Fecal microbiota transplantation in inflammatory bowel disease: beyond the excitement. Medicine 2014;93:e97.

92 Sood A, Mahajan R, Singh A, et al. Role of faecal microbiota transplantation for maintenance of remission in patients with ulcerative colitis: a pilot study. J Crohns Colitis 2019:105

93 Halkjær SI, Christensen AH, Lo BZS, et al. Faecal microbiota transplantation alters gut microbiota in patients with irritable bowel syndrome: results from a randomised double-blind placebo-controlled study. Gut 2018:67:2107-15.

94 Johnsen $\mathrm{PH}$, Hilpüsch F, Cavanagh JP, et al. Faecal microbiota transplantation versus placebo for moderate-to-severe irritable bowel syndrome: a doubleblind, randomised, placebo-controlled, parallel-group, single-centre trial. Lancet Gastroenterol Hepatol 2018:3:17-24.10.1016/\$2468-1253(17)30338-2

95 Holster S, Lindqvist CM, Repsilber D, et al. The effect of allogenic versus autologous fecal microbiota transfer on symptoms, visceral perception and fecal and mucosal microbiota in irritable bowel syndrome. Clin Trans/ Gastroenterol 2019;10:e00034. IBS 1.

96 Ianiro G, Eusebi LH, Black CJ, et al. Systematic review with meta-analysis: efficacy of faecal microbiota transplantation for the treatment of irritable bowel syndrome Aliment Pharmacol Ther 2019:50:240-8.

97 Bajaj JS, Kassam Z, Fagan A, et al. Fecal microbiota transplant from a rational stool donor improves hepatic encephalopathy: a randomized clinical trial. Hepatology 2017;66:1727-38.10.1002/hep.29306 
98 Bajaj JS, Kakiyama G, Savidge T, et al. Antibiotic-associated disruption of microbiota composition and function in cirrhosis is restored by fecal transplant. Hepatology 2018;68:1549-58.

99 Vrieze A, Van Nood E, Holleman F, et al. Transfer of intestinal microbiota from lean donors increases insulin sensitivity in individuals with metabolic syndrome. Gastroenterology 2012;143:913-6.

100 Kootte RS, Levin E, Salojärvi J, et al. Improvement of insulin sensitivity after lean donor feces in metabolic syndrome is driven by baseline intestinal microbiota composition. Cell Metab 2017;26:611-9.

101 Bilinski J, Grzesiowski P, Sorensen N, et al. Fecal microbiota transplantation in patients with blood disorders inhibits gut colonization with antibioticresistant bacteria: results of a prospective, single-center study. Clin Infect Dis 2017;65:364-70.

102 Huttner BD, de Lastours V, Wassenberg M, et al. A 5-day course of oral antibiotics followed by faecal transplantation to eradicate carriage of multidrugresistant Enterobacteriaceae: a randomized clinical trial. Clin Microbiol Infect 2019;25:830-8.

103 Kuijper EJ, Vendrik KEW, Vehreschild MJGT. Manipulation of the microbiota to eradicate multidrug-resistant Enterobacteriaceae from the human intestinal tract. Clin Microbiol Infect 2019;25:786-9.

104 Allegretti JR, Kassam Z, Carrellas M, et al. Fecal microbiota transplantation in patients with primary sclerosing cholangitis. Am J Gastroenterol 2019;114:1071-9.
105 Kang D-W, Adams JB, Gregory AC, et al. Microbiota transfer therapy alters gut ecosystem and improves gastrointestinal and autism symptoms: an open-label study. Microbiome 2017;5.

106 Wang Y, Wiesnoski DH, Helmink BA, et al. Fecal microbiota transplantation for refractory immune checkpoint inhibitor-associated colitis. Nat Med 2018;24:1804-8

107 Routy B, Le Chatelier E, Derosa L, et al. Gut microbiome influences efficacy of PD-1based immunotherapy against epithelial tumors. Science 2018;359:91-7.

$108 \mathrm{Ma} \mathrm{Y,} \mathrm{Liu} \mathrm{J,} \mathrm{Rhodes} \mathrm{C,} \mathrm{et} \mathrm{al.} \mathrm{Ethical} \mathrm{issues} \mathrm{in} \mathrm{fecal} \mathrm{microbiota} \mathrm{transplantation} \mathrm{in}$ practice. Am J Bioeth 2017;17:34-45.

109 Daloiso V, Minacori R, Refolo P, et al. Ethical aspects of fecal microbiota transplantation (FMT). Eur Rev Med Pharmacol Sci 2015;19:3173-80.

110 Davidovics ZH, Michail S, Nicholson MR, et al. Fecal microbiota transplantation for recurrent Clostridium difficile infection and other conditions in children: a joint position paper from the North American Society for pediatric gastroenterology, hepatology, and nutrition and the European Society for pediatric gastroenterology, hepatology, and nutrition. J Pediatr Gastroenterol Nutr 2019;68:130-43.

111 Nicholson MR, Mitchell PD, Alexander E, et al. Efficacy of fecal microbiota transplantation for Clostridium difficile infection in children. Clin Gastroenterol Hepatol 2019:30427-6.

112 Saeedi BJ, Morison DG, Kraft CS, et al. Fecal microbiota transplant for Clostridium difficile infection in a pregnant patient. Obstet Gynecol 2017;129:507-9.

113 ClinicalTrials.gov. Fecal microbiota transplant national registry (FMT). Available: https://ClinicalTrials.gov/show/NCT03325855 [Accessed 1 Jul 2019]. 